\title{
The Condensation of Continuants.
}

By Thomas Muir, LL.D.

1. Rather more than twenty years ago, in a note on this subject, it was shown to the Edinburgh Mathematical Society (Proceedings, II., pp. 16-18) that a special form of continuant, viz., one with univarial diagonals, could be expressed by means of a similar continuant of much lower order. A new mode of proving this theorem, which has lately been hit upon, has unexpectedly led to the discovery that the peculiarity in question is not confined to this special form, but characterises continuants of any form whatever.

2. Taking first a continuant of odd order, viz.,

$$
\left|\begin{array}{ccccccc}
a_{1} & b_{1} & . & \cdot & \cdot & \cdot & \cdot \\
-1 & a_{3} & b_{2} & \cdot & \cdot & \cdot & \cdot \\
\cdot & -1 & a_{3} & b_{3} & \cdot & \cdot & \cdot \\
\cdot & \cdot & -1 & a_{4} & b_{4} & \cdot & \cdot \\
\cdot & \cdot & \cdot & -1 & a_{5} & b_{5} & \cdot \\
\cdot & \cdot & \cdot & \cdot & -1 & a_{6} & b_{6} \\
\cdot & \cdot & . & . & . & -1 & a_{7}
\end{array}\right| \text {, or } \mathrm{K}_{1,7} \text { say, }
$$

and multiplying it by $a_{1} a_{3}{ }^{2} a_{5}{ }^{2} a_{7}$ in the form

$$
\left|\begin{array}{ccccccc}
1 & \cdot & \cdot & \cdot & \cdot & \cdot & \cdot \\
-b_{1} a_{3} & a_{1} a_{3} & a_{1} & \cdot & \cdot & \cdot & \cdot \\
\cdot & \cdot & 1 & \cdot & \cdot & \cdot & \cdot \\
\cdot & \cdot & -b_{3} a_{5} & a_{3} a_{5} & a_{3} & \cdot & \cdot \\
\cdot & \cdot & \cdot & \cdot & 1 & \cdot & \cdot \\
\cdot & \cdot & \cdot & \cdot & -b_{5} a_{5} & a_{5} a_{7} & a_{5} \\
\cdot & \cdot & \cdot & \cdot & \cdot & \cdot & 1
\end{array}\right|
$$


we obtain

$$
\left|\begin{array}{ccccccc}
a_{1} & \cdot & \cdot & \cdot & \cdot & \cdot & \cdot \\
-1 & a_{1} a_{2} a_{3} \mid & b_{2} & -b_{2} b_{3} a_{5} & \cdot & \cdot & \cdot \\
\cdot & \cdot & a_{3} & \cdot & \cdot & \cdot & \cdot \\
\cdot & -a_{1} & -1 & \left|a_{3} a_{4} a_{5}\right| & b_{4} & -b_{4} b_{5} a_{7} & \cdot \\
\cdot & \cdot & \cdot & \cdot & a_{5} & \cdot & \cdot \\
\cdot & \cdot & \cdot & -a_{3} & -1 & \left|a_{5} a_{6} a_{7}\right| & b_{6} \\
\cdot & \cdot & \cdot & \cdot & \cdot & \cdot & a_{7}
\end{array}\right|
$$

where $\left|a_{1} a_{2} a_{3}\right|,\left|a_{3} a_{4} a_{5}\right|,\left|a_{5} a_{6} a_{7}\right|$ are coaxial minors of $\mathbf{K}_{1,7}$, viz.,

$$
\left|\begin{array}{lll}
a_{1} & b_{1} & \cdot \\
-1 & a_{2} & b_{2} \\
. & -1 & a_{3}
\end{array}\right|,\left|\begin{array}{lll}
a_{3} & b_{3} & \cdot \\
-1 & a_{4} & b_{4} \\
. & -1 & a_{5}
\end{array}\right|,\left|\begin{array}{lll}
a_{5} & b_{5} & \cdot \\
-1 & a_{6} & b_{6} \\
. & -1 & a_{7}
\end{array}\right| .
$$

This seven-line determinant, however, is evidently resolvable into

$$
a_{1} a_{3} a_{5} a_{7} \cdot\left|\begin{array}{ccc}
\left|a_{1} a_{2} a_{3}\right| & -b_{2} b_{3} a_{5} & . \\
-a_{1} & \left|a_{3} a_{4} a_{5}\right| & -b_{4} b_{5} a_{7} \\
\cdot & -a_{3} & \left|a_{5} a_{6} a_{7}\right|
\end{array}\right|
$$

consequently there results

$$
\mathrm{K}_{1,7} \cdot a_{3} a_{5}=\left|\begin{array}{ccc}
\left|a_{1} a_{3} a_{3}\right| & b_{3} b_{3} & \cdot \\
a_{1} a_{5} & \left|a_{3} a_{4} a_{5}\right| & b_{1} b_{5} \\
\cdot & a_{3} a_{7} & \left|a_{5} a_{6} a_{7}\right|
\end{array}\right|
$$

When the given continuant is $K_{1,2 n+1}$ its co-fuctor on the left is $a_{i} a_{5} \ldots a_{2 n-1}$, and the continuant on the right is of the $n$th order.

3. For the case where the given continuant is of even order, no separate investigation is necessary, for, putting $a_{7}=1, b_{6}=0$ in the preceding result we have

$$
\mathbf{K}_{1,3 j} \cdot a_{3} a_{5}=\left|\begin{array}{ccc}
\left|a_{1} a_{2} a_{3 j}\right| & b_{3} b_{: 3} & \cdot \\
a_{1} a_{\bar{j}} & \left|a_{3} a_{4} a_{5}\right| & b_{4} b_{5} \\
. & a_{3} & \left|a_{5} a_{6}\right|
\end{array}\right|
$$

the want of symmetry in which at once suggests the alternative result

$$
\mathrm{K}_{1,0} \cdot a_{2,} a_{4}=\left|\begin{array}{ccc}
\left|a_{2} a_{2,}\right| & b_{1} b_{2} & . \\
a_{4} & \left|a_{2} a_{3} a_{4}\right| & b_{33} b_{4} \\
\cdot & a_{6} a_{32} & \left|a_{4} a_{5} a_{6}\right|
\end{array}\right| .
$$


Putting in this last each of the $a$ 's equal to $x$ and each of the $b$ 's equal to $-b c$ we obtain

$$
\mathrm{K}_{1,6} \cdot x^{2}=\left|\begin{array}{ccc}
x^{2}-b c & b^{2} c^{2} & \cdot \\
x & x\left(x^{2}-2 b c\right) & b^{2} c^{2} \\
. & x^{2} & x\left(x^{2}-2 b c\right)
\end{array}\right|
$$

and therefore in the notation of the paper of 1884

$$
\begin{aligned}
\mathrm{F}(b, x, c, 6) & =\left|\begin{array}{ccc}
x^{2}-b c & b^{2} & \cdot \\
c^{2} & x^{2}-2 b c & b^{2} \\
\cdot & c^{2} & x^{2}-2 b c
\end{array}\right| \\
& =\mathbf{F}\left(b^{2}, x^{2}-2 b c, c^{2}, 3\right)+b c \mathrm{~F}\left(b^{2}, x^{2}-2 b c, c^{2}, 2\right) .
\end{aligned}
$$

4. Since from $\left(\mathrm{II}^{\prime}\right)$ we have

$$
\mathrm{K}_{1,8} \cdot a_{2} a_{4} a_{6}=\left|\begin{array}{cccc}
a_{1} a_{2} \mid & b_{1} b_{2} & . & . \\
a_{4} & \left|a_{2} a_{3} a_{4}\right| & b_{3} b_{4} & . \\
\cdot & a_{13} a_{2} & \left|a_{3} a_{5} a_{4}\right| & b_{5} b_{1 i} \\
\cdot & \cdot & a_{8} a_{4} & \left|a_{0} a_{7} a_{8}\right|
\end{array}\right|
$$

it follows on putting $a_{8}=1, b_{7}=0$ that

$$
\mathrm{K}_{1,7} \cdot a_{2} a_{4} a_{65}=\left|\begin{array}{cccc}
\mid a_{1} a_{2} ! & b_{1} b_{2} & . & . \\
a_{4} & \left|a_{2} a_{3} a_{4}\right| & b_{3} b_{4} & . \\
\cdot & a_{6} a_{3} & \left|a_{4} a_{5} a_{6}\right| & b_{5} b_{6} \\
\cdot & . & a_{4} & \left|a_{6} a_{7}\right|
\end{array}\right|
$$

which is an alternative to $(\mathbf{I})$.

5. If now we take identities which give the equivalent of an even-ordered $K$, say $K_{1.8}$, and the equivalent of the differential quotient of this with respect to $a$, viz., the first identity of the preceding paragraph and the identity

$$
\mathrm{K}_{2,8} \cdot a_{4} a_{6}=\left|\begin{array}{ccc}
\left|a_{2} a_{3} a_{4}\right| & b_{3} b_{4} & \cdot \\
a_{6} a_{2} & \left|a_{4} a_{5} a_{6}\right| & b_{5} b_{6} \\
\cdot & a_{4} & \left|a_{6} a_{7} a_{8}\right|
\end{array}\right|,
$$

we obtain at once by division

$$
a_{2} \cdot\left\{a_{1}+\frac{b_{1}}{a_{2}}+\frac{b_{2}}{a_{3}}+\cdot+\frac{b_{7}}{a_{8}}\right\}=\left|a_{1} a_{2}\right|-\frac{b_{1} b_{2} a_{4}}{\left|a_{9} a_{3} a_{4}\right|}-\frac{b_{3} b_{4} a_{6} a_{2}}{\left|a_{4} a_{5} a_{6}\right|}-\frac{b_{5} b_{8} a_{8} a_{4}}{\left|a_{8} a_{7} a_{8}\right|}
$$


The corresponding identity in which the number of $a$ 's is odd is most readily got as before by putting in this $a_{8}=1, b_{7}=0$.

Substituting $x$ for each of the odd-numbered $a$ 's and 1 for each of the even-numbered we find

$$
x+\frac{b_{1}}{1}+\frac{b_{2}}{x}+\frac{b_{3}}{1}+\cdot \ddots_{+\frac{b_{7}}{1}}=x-\frac{b_{1} b_{2}}{x+b_{2}+b_{3}}-\frac{b_{3} b_{4}}{x+b_{4}+b_{5}}-\frac{b_{5} b_{6}}{x+b_{6}+b_{7}}
$$

-a result said to have been first published in 1860 by Heilermann (Zeitschr. f. Math. u. Phys. V. pp. 262-263).

6. Again since we also have

and

$$
\mathrm{K}_{1,8} \cdot a_{3} a_{5} a_{7}=\left|\begin{array}{cccc}
\left|a_{1} a_{2} a_{3}\right| & b_{2} b_{3} & \cdot & \cdot \\
a_{1} a_{3} & \left|a_{3} a_{4} a_{5}\right| & b_{4} b_{5} & \cdot \\
\cdot & a_{3} a_{7} & \left|a_{5} a_{6} a_{7}\right| & b_{6} b_{7} \\
\cdot & \cdot & a_{5} & \left|a_{7} a_{8}\right|
\end{array}\right|
$$

$$
\mathrm{K}_{3,8} \cdot a_{5} a_{7}=\left|\begin{array}{ccc}
\left|a_{3} a_{4} a_{5}\right| & b_{4} b_{5} & . \\
a_{3} a_{7} & \left|a_{5} a_{6} a_{7}\right| & b_{6} b_{7} \\
\cdot & a_{5} & \left|a_{7} a_{8}\right|
\end{array}\right|
$$

it follows by division that

$$
a_{3} \frac{\mathbf{K}_{1,8}}{\mathbf{K}_{3,8}}=\left|a_{1} a_{2} a_{3}\right|-\frac{b_{2} b_{3} a_{1} a_{5}}{\left|a_{3} a_{4} a_{5}\right|}-\frac{b_{4} b_{5} a_{3} a_{7}}{\left|a_{5} a_{6} a_{7}\right|}-\frac{b_{6} b_{7} a_{5}}{\left|a_{7} a_{8}\right|},
$$

- that is to say, we can now express as a continued fraction not only the ratio of $K$ to $\partial K / \partial a_{1}$, but also the ratio of $K$ to $\partial^{2} K / \partial a_{1} \partial a_{2}$.

Further, since

we deduce

$$
\frac{\mathbf{K}_{1,8}}{\overline{\mathbf{K}}_{3,8}}=\frac{\mathbf{K}_{1,8}}{\mathbf{K}_{2,8}} \cdot \frac{\mathbf{K}_{2,8}}{\mathbf{K}_{3,8}}
$$

$$
\begin{aligned}
& \left\{a_{3}\left\{a_{1}+\frac{b_{1}}{a_{2}}+\frac{b_{2}}{a_{3}}+\cdot \cdot+\frac{b_{7}}{a_{8}}\right\} \cdot\left\{a_{2}+\frac{b_{2}}{a_{3}}+\cdot \cdot+\frac{b_{7}}{a_{8}}\right\}\right. \\
& =\left|a_{1} a_{2} a_{3}\right|-\frac{b_{2} b_{3} a_{1} a_{5}}{\left|a_{3} a_{4} a_{5}\right|}-\ddots-\frac{b_{8} b_{7} a_{5}}{\left|a_{7} a_{8}\right|} \text {. }
\end{aligned}
$$


7. From (IV) by putting each of the $b$ 's equal to 1 we have

$$
\left.a_{2}\left\{a_{1}+\frac{1}{a_{2}}+\frac{1}{a_{3}}+\cdot\right\}\right\}=\left(a_{1} a_{2}\right)-\frac{a_{4}}{\left(a_{2} a_{3} a_{4}\right)}-\frac{a_{6} a_{3}}{\left(a_{4} a_{5} a_{6}\right)}-\frac{a_{8} a_{4}}{\left(a_{6} a_{7} a_{8}\right)}-\cdot
$$

where $\left(a_{1} a_{2}\right),\left(a_{3} a_{3} a_{4}\right), \ldots$ are now "simple" continuants. This has a special interest when the continued fraction on the left is the representative of a quadratic surd. Knowing, for example, that

$$
\sqrt{13}=3+\frac{1}{1}+\frac{1}{1}+\frac{1}{1}+\frac{1}{1}+\frac{1}{6}+\ldots
$$

we conclude from it that

$$
\sqrt{13}=4-\frac{1}{3}-\frac{6}{13}-\frac{1}{13}-\frac{6}{3}-\frac{1}{8}-\ldots
$$

The convergents to the former continued fraction are

$$
3,4,3 \frac{1}{2}, 3 \frac{2}{3}, 3 \frac{3}{5}, 3 \frac{20}{3}, 3 \frac{23}{3}, 3 \frac{43}{11}, \ldots
$$

and to the latter

$$
4, \quad 3 \frac{2}{3}, \quad 3 \frac{20}{3}, \quad 3 \frac{4}{4}, \ldots
$$

the $r$ th convergent in the second case being the same as the $2 r$ th convergent in the first.

The process of condensation may, of course, be continued indefinitely. In the case of $\sqrt{13}$ the next result in order is

$$
\sqrt{13}=\frac{11}{3}-\frac{26}{426}-\frac{144}{251}-\frac{1014}{226}-\frac{144}{476}-\frac{234}{51}-\ldots
$$

where the lst, 2 nd, 3rd, .. convergents are the 4 th, 8 th, 12 th, ... of the original continued fraction. 